
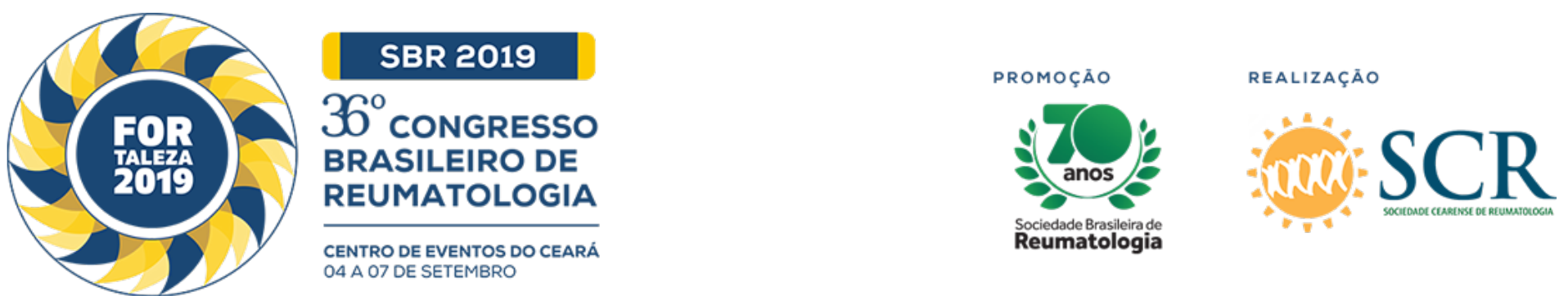

\title{
QUALITY OF LIFE AND FUNCTIONAL CAPACITY OF RHEUMATOID ARTHRITIS PATIENTS TREATED WITH BIOLOGICAL VERSUS CONVENTIONAL DISEASE-MODIFYING ANTIRHEUMATIC DRUGS : OVERVIEW OF SYSTEMATIC REVIEWS
}

Gustavo Fogolin Rosal (HCFMUSP, São Paulo, SP, Brasil), Moacyr Roberto Cuce Nobre (InCor-HCFMUSP, São Paulo, SP, Brasil)

\section{BACKGROUND}

Several randomized clinical trials (RCT) have been conducted in recent years on the efficacy of biological disease-modifying antirheumatic drugs (bDMARD) in the treatment of rheumatoid arthritis (RA). However, systematic reviews (SR) on this topic still raise doubts about the real efficacy related to functional capacity and quality of life. This is an overview that summarizes the evidences generated by SR of RCT that compare treatments using bDMARD versus conventional disease-modifying antirheumatic drugs (CDMARD), considering functional capacity and quality of life of the patients with RA, as well as evaluating the methodological quality of the SR that have been published on this subject.

\section{MATERIALS AND METHODS}

In this overview of the systematic reviews, we have followed the directives contained in Cochrane Handbook for Systematic Reviews of Interventions Version 5.1.0 (updated March 2011) and in Preferred Reporting Items for Systematic Review and Meta-Analysis Protocols (PRISMA-P). Databases from PubMed (Medline), EMBASE and Cochrane were used for obtaining SR with or without meta-analyses of RCT that assessed HAQ and SF-36 in RA patients treated with bDMARD compared to CDMARD. Two researchers, performing independently, conducted the data selection and extraction from the SR, evaluated their methodological quality using Assessment of Multiple Systematic Reviews (AMSTAR), and classified the quality of evidences using Grading of Recommendations Assessment, Development and Evaluation (GRADE). This overview of the systematic reviews has been registered in the PROSPERO database (http://www.crd.york.ac.uk/PROSPERO/).

\section{RESULTS}

The greater part of the 7 systematic reviews and meta-analyses of RCT included in this overview presented a high methodological quality as evaluated by AMSTAR, and the quality of evidences from SR varied between low and high quality as per GRADE. Functional capacity improvement and quality of life observed in the initial treatment period ( 24 weeks) demonstrated being higher when using bDMARD therapy. This difference between treatments was not noticed in the long term ( 52 weeks).

\section{CONCLUSION}

Evidences that varied between low and high quality demonstrated that the BDMARD agents presented improvements on functional capacity and quality of life during the initial treatment period ( 24 weeks) when compared to CDMARD. However, there are no differences in the long term treatment period (52 weeks) between the use of bDMARD therapy and CDMARD therapy. 